\title{
Clinical study \& laborotory profile of rickettsial fever in children- a study from rural Maharashtra
}

\author{
Sunil S. Vaidya ${ }^{1}$, Atul A. Kulkarni ${ }^{2}$ \\ ${ }^{1}$ Professor, Department of Pediatrics, Ashwini Rural Medical College, Hospital \& Research Centre, Kumbhari, Solapur \\ (Maharashtra), ${ }^{2}$ Assistant Professor, Department of Pediatrics, Ashwini Rural Medical College, Hospital \& Research \\ Centre, Kumbhari, Solapur, Maharashtra, India.
}

Address for Correspondence: Dr Sunil S. Vaidya, Email: drsvaidya @ gmail.com

\begin{abstract}
Introduction: Rickettsial fever has been reported to be endemic in the Himalayan belt, Maharashtra and Karnataka in India among the adult population. Pediatric data on the same is limited in developing countries. Recently, the profile of rickettsial fever has been described in children in South India with similar clinical features. Material Methods: This study was conducted from the patients admitted in our hospital, Ashwini Rural Medical College, Hospital \& RC, Solapur, from the month of January 2014 to June 2015. The inclusion criteria were, clinical suspicion \& supportive lab evidence - Weil Felix, positive leucocytosis, thrombocytopenia. Results: In our study age of presentation ranged from 6 months to 12 years, with mean age of 7 yrs, there was no statistically significant sex difference. All patients presented with fever \& purpuric rash was seen in $82 \%$, altered sensorium was seen in $58 \%$, seizures were seen $34 \%$ \& Hepatosplenomegaly was seen in $65 \%$ of cases. Other investigations: In our study CSF examination was done in 25 patients of which 10 had abnormal findings, 6 showed low sugar and 8 high protein. In our study according to the Weil Felix titers, most probable disease would be tick borne spotted fever or epidemic typhus, since no louse infestation (the scalp and body infestation, lymphadenopathy) was seen in any of the patients, and most of them were from rural areas more chances of tick infestation. Conclusions: The diagnosis of rickettsia should always be kept in mind for workup of exanthematous fever. High index of clinical suspicion and good laboratory co- relation are helpful in detection of more no of cases. Early diagnosis and treatment with doxy and chloramphenicol can reduce the hospital stay and cost. Associated mixed infections may mislead diagnosis and are more fatal. Weil Felix test is not diagnostic standard. It should be interpreted in good clinical context, still it is easily available to all \& remains good screening test.
\end{abstract}

Key words: Rickettsial Fever, Weil Felix Test, Rash, Hepatosplenomegaly.

\section{Introduction}

Microorganisms belong to the family of rickettsiaceae and are obligate intracellular cocco-bacilli [1]. The causative organism was named after Howard Ricketts, who was the first to demonstrate the role of the tick (Dermacentor andersonii) as the vector for the disease in western Montana in the US in 1906 [2]. The illnesses caused can be divided into 3 main biogroups - Spotted fever, typhus and scrub typhus groups [3]. The most frequent presenting symptoms of the illness include fever, headache, rash, and myalgias [4]. Rickettsial fever has been reported to be endemic in the Himalayan belt, Maharashtra and Karnataka in India among the

Manuscript received: $24^{\text {th }}$ June 2016

Reviewed: $4^{\text {th }}$ July 2016

Author Corrected; $15^{\text {th }}$ July 2016

Accepted for Publication: $30^{\text {th }}$ July 2016 adult population [5]. Pediatric data on the same is limited in developing countries. Recently, the profile of rickettsial fever has been described in children in South India with similar clinical features [6].

Rickettsial fever is an acute febrile zoonotic disease spread by bites of ticks and mites. Rickettsiae make up a family of gram - ve coccobaccilli and short bacilli that grow strictly in eukaryotic cells. They are obligate intracellular parasites.

Humans are accidental host. The family rickettsiae is named after Howard Taylor Ricketts who discovered spotted fever and died during his studies (1909). The family has been classified in 4 genera as Rickettsia, Coxiella, Rochemalia \& Erhlichia [7], [8]. 
Rickettsia have varied clinical spectrum of manifestations, including the CNS, RS, and GIT. Early Clinical diagnosis with high index of suspicion can prevent morbidity \& mortality.

Prevalence of this disease is worldwide and in recent times increased incidence in India. The varied clinical spectrum, lack of clinical suspicion, absence of adequate laboratory techniques, expensive tests, all these pose a great challenge in diagnosis and treatment [9], [10].

Rickettsial disease can be dangerous if missed. We have notice increased incidence in the past 10 years. Thus we conducted a study to evaluate the clinical data of patients suffering from it and admitted to our hospital.

This study aims to increase clinical suspicion, awareness about laboratory evaluations, and treatment of rickettsial infections.

Type of study: Retrospective study

Methodology: This study was conducted from the patients admitted in our hospital, Ashwini Rural Medical College, Hospital \& RC, Solapur, from the month of January 2014 to June 2015.

\section{The inclusion criteria were:}

1. clinical suspicion.

2. supportive lab evidence - Weil Felix, positive leucocytosis, thrombocytopenia,

- Clinical Suspicion was based on history of fever, non confluent maculopapular or purpuric rash involving palms and soles, and neurological symptoms.

- Weil Felix test for (OX-19, 0X-2, 0X-K strains) was done on each patient of clinical suspicion. It is a slide agglutination test done according to manufacturer's instructions, from Plasmatech laboratories, Bridfort, UK. The kit tests serum dilutions from 1:20 to 1:320. Significant titre is $1: 80$, those with positive titre were included in our study.

On admission, data of age, sex, local residing area, exposure to animals, etc was recorded and, -complete blood count, -malarial parasite, -urine exam. was done on all patients. CSF, electrolytes, chest -X-ray, USG, dengue IgM, CT scan done as and when needed. All patients were treated with: chloramphenicol (100 $\mathrm{mg} / \mathrm{kg} /$ day) in 3 divided doses.or doxycycline (5 $\mathrm{mg} / \mathrm{kg} /$ day) as single dose or in some cases both drugs were given.

\section{Results}

In our study of 9 months period, 60 patients satisfied our inclusion criteria, age ranged from 6 months to 12 years, maximum incidence in 2 to 7 years age group (70\%), and male to female ratio was 1.2:1.

Table No-1: Major presenting symptoms.

\begin{tabular}{|c|c|c|c|}
\hline Sr. & Clinical features & No. of cases & Percentage \\
\hline 1 & Fever & 60 & 100 \\
\hline 2 & Hepatosplenomegaly & 39 & 65 \\
\hline 3 & GI upset & 15 & 25 \\
\hline 4 & Convulsions & 20 & 34 \\
\hline 5 & Altered sensorium & 35 & 10 \\
\hline 6 & Pain in legs & 6 & 82 \\
\hline 7 & Purpuric rash & 49 & 7 \\
\hline 8 & Upper GI bleeding & 4 & 5 \\
\hline 9 & Pneumonia & 3 & \\
\hline
\end{tabular}

Table No-2: Investigations.

\begin{tabular}{|c|c|c|c|}
\hline INV & Value & No & \% \\
\hline Mean HB & $9.3 \%$ & & 66 \\
\hline Leucocytosis & $>10,000$ cell $/ \mathrm{mm}$ & 40 & 56 \\
\hline Thrombocytopenia & $>100,000$ & 33 & \\
\hline
\end{tabular}

CBC-Mean Hb was $9.3 \mathrm{gm} / \mathrm{dl}$; Leucocytosis (>10,000 cells/mm) in 66\%, thrombocytopenia (<1 lakh) was seen in $56 \%$. CSF analysis done in 25 patients of which 10 were abnormal, sugar low in 6 cases, proteins high in 8 cases, pleocytosis in all cases with mean cell count 78 cells $/ \mathrm{mm}$. 
Table No-3: CSF analysis.

\begin{tabular}{|c|c|c|}
\hline CSF feature & No & \% \\
\hline Sugar low & 6 & 10 \\
\hline Protein high & 8 & 13 \\
\hline Pleocytosis & 60 & 100 \\
\hline
\end{tabular}

Other lab parameters:

Coagulation studies-PT.APTT was prolonged in 5 out of 20 cases, FDP D Dimer was positive in 4 cases.

Echocardiography - was done in 10 cases exhibiting tachycardia with gallop rhythm, 5 of them showed myocardial involvement in form of reduced $\mathrm{EF}<50 \%$.

CT/MRI- scan was done in 18 patients, of which 8 were normal, 7 had cerebral edema, and 3 with features of meningitis.

Outcome- 5 children required mechanical ventilation, out of it 3 expired and 2 recovered well. Responses to doxy, chloramphenicol was quite good and most were afebrile by 48-72 hours. Out of total 60 cases 55 (92\%) recovered well, $7 \%$ expired and 3 cases went AMA.

\section{Discussion}

Rickettsial diseases are an important but often under recognized cause of febrile illness locally. Of the wide range of rickettsial diseases, typhus disease is the most commonly recognized entity in our area.

In our study age of presentation ranged from 6 months to 12 years, with mean age of 7 yrs, there was no statistically significant sex difference. This is similar to Colomba et. al [11] \& Nigwekar P et. a 1 [12] who showed median age of $5 y$ rs and 6 yrs respectively with no significant sex deference.

Majority of patients presented with fever (100\%), purpuric rash was seen in $82 \%$, which is similar to Colomba et. al [11] \& Nigwekar P et. al [12]. Altered sensorium was seen in $58 \%$ which is much more as compared with Mahajan et. al[13]. (24\%) \&. Seizures were seen $34 \%$ in comparison to Mahajan et. al[13]. (19\%) \& Nigwekar $P$ et. al [12]. (36\%). Hepatosplenomegaly was seen in $65 \%$ as compared with Mahajan et. al [13]. (43\%) \& Nigwekar P et. al [12]. (34\%).

Other investigations: In our study CSF examination was done in 25 patients of which 10 had abnormal findings, 6 showed low sugar and 8 high protein. This proves CNS involvement in rickettsial fever.

In our study according to the Weil Felix titers, most probable disease would be tick borne spotted fever or epidemic typhus, since no louse infestation (the scalp and body infestation, lymphadenopathy) was seen in any of the patients, and most of them were from rural areas more chances of tick infestation. Hence tick borne spotted fever is most likely cause but still further definitive investigations like PCR should be done to detect the different rickettsial organisms.

Weil Felix test still remains the most commonly used serological test. It may give false positive reactions with Proteus sp., Leptospirosis, Borrelia and severe liver disease. It is negative for R.pox, R. quintana, with brillzinsser disease. Even though sensitivity and WF test is low there are several reports which suggest good corelation of it with clinical suspicion and other tests. Immunoflourence Assay is taken as gold standard test as it is most sensitive and most specific, but it is too costly for us and even not available easily.

The patients with late presentation were in altered sensorium, with predominant neurological features. They had poor outcome as compared to those who had received doxycycline prophylactically.

\section{Conclusions}

Rickettsial fever does exist in our area and its incidence is rising. The diagnosis of rickettsia should always be kept in mind for workup of exanthematous fever. High index of clinical suspicion and good laboratory corelation are helpful in detection of more no of cases. Early diagnosis and treatment with doxy and chloramphenicol can reduce the hospital stay and cost. Associated mixed infections may mislead diagnosis and 
are more fatal. Weil Felix test is not diagnostic standard. It should be interpreted in good clinical context, still it is easily available to all \& remains good screening test.Use of empirical treatment may be considered to reduce the morbidity and mortality observed with this disease.

\section{Funding: Nil, Conflict of interest: Nil \\ Permission from IRB: Yes}

\section{References}

1. Walker DH. Rickettsiae and rickettsial infections: the current state of knowledge. Clin Infect Dis. 2007 oct; 45 1:S39-44

2. Ricketts HT. A micro-organism which apparently has a specific relationship to Rocky Mountain spotted fever. JAMA 1909;52: $379-380$

3. Jensenius M, Fournier P, Raoult D. Rickettsioses and the international traveler. Clin Infect Dis. 2004; 34 (10):1493-9

4. Helmick CG, Bernard KW, D'Angelo LJ. Rocky Mountain spotted fever: clinical, laboratory, and epidemiological features of 262 cases. J Infect Dis. 1984 Oct;150(4):480-488.

5. Padbidri VS, Gupta NP. Rickettsiosis in India: A review. J Indian Med Assoc 1978; 71: 104-107.
6. N. Murali, Swathi Pillai, Thomas Cherian, P. Raghupathy, V. Padmini, Elizabeth Mathai. Rickeetsial infections in South India: How to spot the spotted fever. Indian Pediatrics 2001; 38: 1393-1396.

7. Raoult D, Parola P, editors. Rickettsial Diseases. New York: Informa Healthcare USA 2007.

8. Frieden IJ, Resnick SD. Childhood exanthems - old and new. Pediatr Clin N Am. 1991; 38: 859-887.

9. Reller ME, Dumler SJ. Nelson text book of pediatric. $19^{\text {th }}$ ed. Epidemic Typhus (Rickettsia prowazekii); 222.2: 1047-8.

10. Rathi N.,Rathi A. Indian Pediatrics: 2010 Feb; 147:159.

11. Colomba C, Laura S, Valentino FP, Raffaella R, Lucio T. Mediterranean spotted fever: clinical and laboratory characteristics of 415 Sicilian children. BMC Infect Dis. 2006;6: 60.

12. Nigwekar P, Kavar Y, Shrikhande DY, Ashok Kumar C. Clinico-pathological profile of Rickehsial Fever in a rural area of western Maharashtra, India. Pravara Med Rev. 2013;5(3):5-9.

13. Mahajan SK. Scrub typhus. J. Assoc. of Physic India. 2005;53:954-58.

\section{How to cite this article?}

Sunil S. Vaidya, Atul A. Kulkarni. Clinical study \& laborotory profile of rickettsial fever in children- a study from rural Maharashtra. Int J Pediatr Res.2016;3(8):553-556.doi:10.17511/ijpr.2016.i08.02 